\title{
Correction to: An Improved Vehicle Detection and Tracking Model
}

Libin $\mathrm{Hu}$, Zhongtao Li, Hao Xu, and Bei Fang

\author{
Correction to: \\ Chapter "An Improved Vehicle Detection and Tracking \\ Model" in: X. Zeng et al. (Eds.): International Symposium \\ for Intelligent Transportation and Smart City (ITASC) 2019 \\ Proceedings, SIST 127, \\ https://doi.org/10.1007/978-981-13-7542-2_8
}

In the original version of the book, belated affiliation corrections in the chapter "An Improved Vehicle Detection and Tracking Model" for all the authors have been incorporated as indicated below:

1. School of Information Science and Engineering, University of Jinan, Jinan 250022, China

2. Shandong Provincial Key Laboratory of Network Based Intelligent Computing, University of Jinan, Jinan 250022, China 